pairment during life. Had the diagnosis been made during life and the tumour resected, there is a strong presumption in the light of Stauffer's experience that complete resolution of the abnormal features may have occurred. This is important as many such patients are probably refused operative treatment at present on the grounds that the abnormality of functional efficiency of the liver denotes a disseminated lesion. The present case emphasises the need to undertake pyelographic examination in patients presenting with unexplained hepatomegaly with or without splenic enlargement and liver cell dysfunction and raises the optimistic note that even a severe degree of functional impairment of the liver need not necessarily be a bar to surgical treatment in that complete resolution of the abnormal features may follow a successful nephrectomy.

Epileptiform seizures in the absence of a demonstrable intracranial abnormality, as in this case, should probably also be regarded as a nonmetastatic lesion likely to resolve after successful removal of the primary malign ing by recent reviews of nonmetastatic manifestations of malignant disease (Pinals and Krane, 1962; Greenberg and others, 1964), epilepsy cf this type does not appear to have been described previously.

\section{Summary}

A case is described of a woman of 47 who suffered from a fatal illness for 18 months, characterised by constitutional disturbance, weight loss, fever, non-specific anaemia, progressive hepatosplenomegaly and increasing liver cell dysfunction and preterminally grand mal epilepsy. Autopsy, which revealed a hyperne- phroma of the left kidney, failed to show macroscopic or histological abnormality of the liver, brain or the intracranial vasculature. It ts suggested that this was a case of nonmetastatic hepatosplenomegaly and epilepsy in whom res solution of these features could conceivably have occurred if the renal tumour had been identifieg and resected during life. Further information on. the frequency of such manifestations would bs helpful in the diagnosis and management of rena carcinoma.

I am grateful to Professor A. G. Macgregor fo permission to study this case who was admitted under his care and to Professor A. C. Currie fo the post-mortem examination.

\section{REFERENCES}

BotTIGER, L. E. (1960a): Studies in Renal Carcinoma I. Clinical and Pathologic Anatomical Aspects Acta med. scand., 167, 443.

, (1960b): Studies in Renal Carcinoma : II. Biøe chemical Investigations, Ibid, 167, 455.

CreEvy, C. D. (1935): Confusing Clinical Manifesta tions of Malignant Renal Neoplasms, Arch. interr. Med., 55, 895.

GreenberG, E., Divertie, M. B., and WoOlner, L. $\overrightarrow{\mathrm{B}}$ (1964): A Review of Unusual Systemic Manifestzo tions Associated with Carcinoma, Amer. J. Med 36, 106.

Melicow, M. M., and Ulson, A. C. (1960): Noro urologic Symptoms in Patients with Renal Cancer, J. Amer. med. Ass., 172, 146.

Pinals, R. S., and KRANe, S. M. (1962): Mediç Aspects of Renal Carcinoma, Postgrad. med 38, 507.

STAUFFER, M. H. (1961): Nephrogenic Hepatosplèn megaly, (Abstract), Gastroenterology, 40, 694.

Weinstein, E. C., Geraci, J. E., and Green, L. (1961): Hypernephroma Presenting as Fever \& Obscure Origin, Proc. Mayo Clin., 36, 12.

\title{
TWO PATIENTS WITH CARDIAC MYXOMA:-ONE PRESENTING AS BACTERIAL ENDOCARDITIS, AND ONE AS CONGESTIVE
CARDIAC FAILURE
}

\author{
ANGUS RAE, M.B., M.R.C.P., M.R.C.P.E. \\ Senior Medical Registrar, The London Hospital, E.1.
}

TUMOURS of the heart may be primary or secondary, the latter being 20-40 times commoner than the former (Prichard, 1951). Thorel in 1903 found no primary tumours in a series of 3,300 autopsies. Ravid and Sachs (1943) found one such tumour in every 1,888 consecutive autopsies and Straus and Merliss (1945) reported 18 in 480,331 autopsies. Of the primary tumours nearly half are myxomata.
The clinical manifestations of these tumours ares protean, and as with other conditions which are uncommon the diagnosis is sometimes not made because it is not considered. Even when cort sidered it is not always easy to establish. This unfortunate because although most myxomata may be removed with comparative safety, if they are not, once symptoms have begun, death wif follow sooner or later (Goodwin, 1963). 


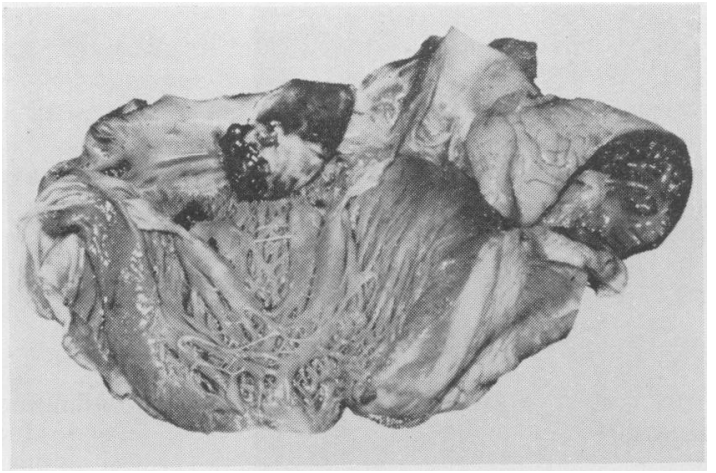

FIG. 1. The tumour from Case 1 lying in the left atrium and attached to its wall covered with blood clot.

The two cases described here are of cardiac myxomata diagnosed at post mortem. One presented the clinical picture of bacterial endocarditis, and the other of congestive cardiac failure.

\section{Case No. 1}

A 48-year-old man presented in December 1959 with a four-week history of fever and rigors. These had followed a few days after an internal urethrotomy had been performed for urethral stricture.

On examination he was febrile and sweating. The first heart sound was louder than normal and there was a soft mid-diastolic murmur in the mitral area. This murmur varied in intensity from day to day; occasionally it could not be heard. Clubbing of the fingers was present. There were no other abnormal physical signs.

Investigations: Hb. 11.1 g. \%, WBC, 11,000/cu. mm. $83 \%$ polys., ESR $60 \mathrm{~mm} . / \mathrm{hr}$. (Westergren); urine: many leucocytes, culture grew streptococcus faecalis; chest X-ray and ECG within normal limits; blood culture grew streptococcus faecalis.

A diagnosis of mitral stenosis with bacterial endocarditis was made. The patient was treated with intramuscular streptomycin and penicillin. Despite increasing doses of these antibiotics the fever and rigors continued. Following one of these rigors there was a sudden onset of right upper motor neurone hemiparesis with dysphasia. Fourteen days after commencement of treatment and again following a rigor there was sudden severe abdominal pain. Rigidity and absent bowel sounds led to a diagnosis of mesenteric embolus with perforation of gangrenous bowel. Laparotomy was performed, and revealed multiple bowel perforations. These were sutured and a drainage tube inserted. The patient died the following day of generalised peritonitis.

Necropsy revealed a pedunculated myxoma $4 \mathrm{~cm}$. $x 2 \mathrm{~cm}$. (Fig. 1) hanging from the wall of the left atrium. It was covered with blood clot, fibrin and vegetations. The mitral valve was normal. Histology showed that organisms were invading the tumour. The superior mesenteric artery was blocked with ante mortem blood clot. Many perforations of gangrenous bowel were present. There was generalised peritonitis. There were recent septic infarcts in the left kidney and spleen.

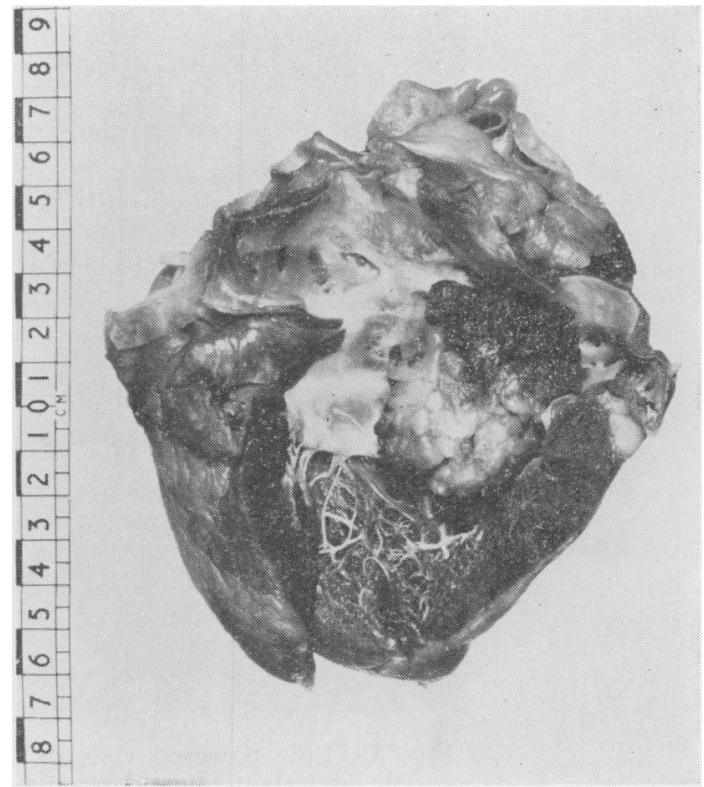

FIG. 2. The tumour from Case 2 is seen lying in the left atrium obstructing the mitral orifice.

Case No. 2

A 60-year-old coloured woman of Indian extraction presented in July 1960 with a four-month history of increasing exertional dyspnoea. She had suffered several attacks of nocturnal dyspnoea during the preceding two months. There had been swelling of the ankles in the evenings. She had occasional palpitations but no fainting attacks or loss of consciousness.

On examination there was biventricular enlargement. Pulse regular, small volume, BP $110 / 70 \mathrm{~mm}$. H.g. JVP raised $2 \mathrm{~cm}$. There was mild pitting oedema of the ankles and the liver edge was palpable and tender. Crepitations were heard over both lung bases. Auscultation revealed a third heart sound and a loud closely split second sound at the pulmonary area. There were no other abnormal physical signs.

Investigations: Hb. 14.3 g. \%, WBC 6,800/cu. mm., normal differential count; the ESR varied between 15 and $77 \mathrm{~mm}$./ hr. (Westergren); LE cells were not seen. Serum albumin levels were normal but there was a persistent mild increase in the gamma globulin. SGOT, liver function tests and serum electrolytes were all normal. ECG showed evidence of increasing left atrial enlargement (P mitrale in standard leads). Chest X-ray showed varying degrees of pulmonary oedema and in the closing phase of the illness, bilateral pleural effusions. The cardiac shadow was increased in size. Barium swallow did not demonstrate an enlarged left atrium.

A diagnosis by exclusion of cardiomyopathy of unknown cause was made. This diagnosis received some support from the raised ESR and gamma globulin. Digitalis, diuretics, salt restriction and later steroids brought only temporary relief of symptoms. The pattern of improvement and relapse continued and the patient died of irreversible pulmonary oedema seven months after admission to hospital.

Necropsy revealed an enlarged heart weighing 


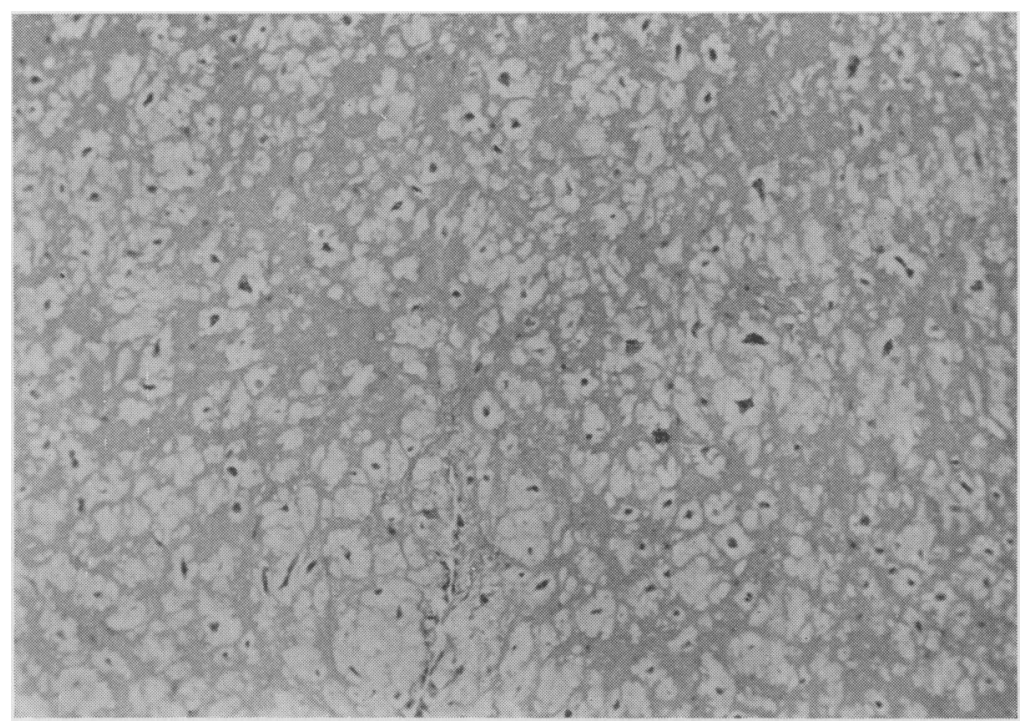

FIG. 3.-Low powered view of tumour from Case 1 to show stellate cells in abundant mucoid stroma. X 63, H \& E stain.

$460 \mathrm{~g}$. The right ventricle was enlarged. There was a tumour of gelatinous consistency measuring $4 \times 3 \times 3$ $\mathrm{cm}$. lying in the left atrium attached by a small pedicle to its posterior wall (Fig. 2). There were a few small blood clots on the surface of the tumour. The lungs were congested and there were bilateral pleural effusions. The liver and to a lesser extent the spleen were congested.

\section{Comment}

The histology of both tumours showed considerable areas of necrosis, but in the better preserved portions, the typical histological appearances of myxoma were apparent (Fig. 3).

In these two patients the diagnosis of cardiac myxoma was made at necropsy. In the iirst case, fever, apparent valvular lesion and positive blood culture following pelvic manipulations led to a diagnosis of bacterial endocarditis. The organisms having gained entrance to the blood stream infected the tumour in the same way as they might have infected a diseased valve.

This is an unsual occurrence having been only once previously reported (Dick and Mullin, 1956). In their patient septicaemia was caused by staphylococcus aureus. Erythromycin and streptomycin eradicated this infection, but the patient relapsed and candida parapsilosis was then cultured from the blood stream. At necropsy this fungus was found growing on a myxoma in the left atrium. It is probable that the septicaemia and later the mycohaemia were perpetuated by organisms growing on the tumour. No portal of entry was found for the original infection.

In the second case, the differential diagnosis lay between ischaemic heart disease and cardiomyopathy. There was no history of angina and no ECG change to support the former diagnosis.
Accordingly a diagnosis of cardiomyopathy wa: made. The rise in ESR and gamma globulin wers considered to be due to some unidentified chromig inflammatory process giving rise to this conditen Suspicion of the correct diagnosis was हैot aroused. In retrospect, had cardiac myxoma bese considered, the raised ESR and gamma globuli would equally have lent support to this diagnosis as to cardiomyopathy (MacGregor and Cullers 1959).

\section{Discussion}

Campeau and David (1960) in an analysis of $6 \frac{3}{3}$ cases of cardiac myxoma found that 57 were in the left atrium and 8 in the right. The youngest patient was one month and the oldest 73 years The average age was 45 years. The average dura tion of symptoms was one year, the shortest one month, the longest 30 years. In this series the sex incidence was similar. Goodwin (1963), howeveg found twice as many women as men in a series of 45 left atrial myxomata.

The tumours may measure up to $10 \mathrm{~cm}$ ? (Prichard, 1951) and are usually pedunculated They may be smooth or lobular and are occasionally villous. There is often thrombus on their surfaces, and at one time they were not thought to be tumours, but organising thrombus (Husten, 1922). However, evidence has been prof duced that they are neoplasms by Fisher and Helb strom (1960), and it has been pointed out that the hearts in which they occur are otherwise normal and that there is no reason for thrombus to form in them. The tumours may occasionally calcify (Goodwin, 1963).

Tumours in the left atrium are frequently of a pedicle and attached near the rim of the foss 
ovalis. Prichard suggests that the buffetting they receive in the atrium might account for their myxoid characer.

Left atrial tumours produce symptoms when they grow large enough to obstruct the venous return to the atrium or the mitral orifice. Symptoms may be intermittent, often varying with posture, or there may be progressive unremitting cardiac failure. Syncope or pulmonary oedema may occur if a pedunculated tumour floats into and obstructs the mitral orifice. The symptoms may improve dramatically with change in posture. In the majority of cases murmurs are heard and these may also change with posture. The auscultatory signs of mitral stenosis or less commonly incompetence may be present. Obstructive and later vasoconstrictive pulmonary hypertension is often found with enlargement of the right ventricle, loud pulmonary second sound, pulmonary ejection clicks and murmurs, and increase in jugular venous ' $a$ ' wave. It has been shown that there is a decrease in the blood flow to the lower lobes of the lungs in left atrial myxoma, similar to that found in mitral stenosis (Dollery, West, Goodwin, Hugh-Jones and Wilckens, 1961). Some of these cases have been misdiagnosed as corpulmonale or as pulmonary hypertension due to multiple pulmonary emboli. A patient with a leiomyosarcoma of the left superior pulmonary vein growing into the left atrium was described by Kidd, Carson, and Lamont (1961). She presented with exertional dyspnoea and haemoptysis. Cardiac catheterisation showed a normal wedge pressure in the right lung field. This was thought to exclude mitral stenosis or a left atrial myxoma. Had the wedge pressure been taken in the left upper lobe, as the authors suggest, the diagnosis might have been established. This point should be remembered when investigating patients with pulmonary hypertension or haemoptysis. In many cases left atrial myxomata have been discovered when surgery has been performed following a mistaken diagnosis of mitral stenosis. The shorter history, changing quality of the murmur from time to time and with position, and the absence of atrial fibrillation are typical of myxoma.

Tumours of the right atrium may obstruct the tricuspid valve giving rise to right heart failure with oedema, ascites and hepatomegaly. The physical signs of tricuspid stenosis or incompetence or of constrictive pericarditis may be present and may change with posture.

Two patients with right atrial myxoma and polycythaemia have been reported (Levinson and Kincaid, 1961; Siggillino, Crawley, Clauss, Reed, and Tice, 1963). The reason for this association is not clear but Siggillino suggests that prolonged tissue hypoxia might stimulate increased red cell production.

Peripheral emboli may arise either from thrombus on the surface of the tumour, or may consist of myxomatous material itself (Bland, 1957). Cumming and Finkel (1961), have described a case where at autopsy the left internal carotid, the left middle cerebral, the right renal, the splenic and other smaller arteries all contained myxomatous material. The tumour extended through the foramen ovale and pulmonary emboli occurred causing recurrent pleuritic pain. They record that of five cases of left atrial myxoma in children, four had cerebral emboli. This is an important diagnostic clue, as cerebral emboli are rare in children. Occlusion of the central artery of the retina due to embolus in left atrial myxoma was described by Mills and Philpott (1951). Splinter haemorrhages and purpura may also be seen. Pulmonary emboli arise from tumours of the right atrium and if multiple, may cause pulmonary hypertension and further complicate the clinical picture. Pulmonary thrombosis in situ in left atrial tumours is related to pulmonary vascular disease. In addition decreased cardiac output in left atrial tumours is associated with increased incidence of leg and pelvic vein thrombosis, and therefore of pulmonary embolus.

MacGregor and Cullen (1959) have drawn attention to the occurrence of fever, anaemia and a raised ESR in a number of cases. Mild increases in gamma globulin may be present. In the presence of a cardiac murmur, bacterial endocarditis will be considered, but blood cultures are negative and the spleen does not enlarge. Lekisch (1957) suggests that this clinical picture is caused by degenerative changes in the tumour producing a febrile reaction.

Lastly, angina of effort may occur due to low cardiac output and cardiac infarction has been reported due to emboli (Harvey, 1959).

Having suspected the tumour on clinical grounds, the diagnosis may be difficult to establish. Right heart catheterisation shows elevated pulmonary pressure in left atrial tumours indicating impaired pulmonary venous return. Change in the pressure level due to shifting position of the tumour was described by Ellis, Mankin, and Burchell (1958). Similarly in right heart tumours elevated right atrial pressure tracings may occur, suggesting tricuspid stenosis or incompetence. Again the findings may change with movement of the tumour.

The best way to demonstrate the tumours is by angiocardiography (Gravier and Schlienger, 1960) when a lobulated filling defect in the atrium is seen. In left atrial tumours this filling defect may be seen to oscillate between atrium and ventricle (Goldberg, Glenn, Dotter, and Steinberg, 1952; Steinberg, Dotter, and Glenn, 1953; Goodwin, Stanfield, Steiner, Bentall, Sayed, Bloom, and Bishop, 1962). However, apparent filling defects may be seen due to blood from pulmonary veins diluting the contrast medium in the left atrium. A deep left atrial impression was seen on the oesophagogram by MacGregor and Cullen.

Once diagnosed, the tumour should be removed. The first successful removal of such a tumour is credited to Crafoord of Stockholm in 1954. Dugan 
and Walstad (1960) record a successful removal and mention 15 other cases. Since this time many more have been reported. Dugan and Walstad suggest that removal be carried out either under hypothermia or by employing extra-corporeal circulation. Should the patient be operated on for mitral stenosis and a tumour be found it should be removed during a planned operation at a later date.

\section{Conclusion}

Cardiac myxoma is a rewarding ante-mortem diagnosis. It should be considered in atypical cases of mitral and tricuspid disease, especially when the murmurs change from time to time or with unexplained pulmonary hypertension and haemoptysis, progressive cardiac failure without obvious cause, emboli in the absence of atrial fibrillation or pyrexia of undetermined origin.

\section{REFERENCES}

Bland, E. F. (1957): Case Records of the Massachusetts General Hospital. In discussion, New Engl. J. Med., 256, 516.

Campeau, L., and David, P. (1960): Myxoma of the Heart, Canad. med. Ass. J., 82, 586.

C.RAFOORD, C. (1955): In Henry Ford Hospital International Symposium on Cardiovascular Surgery; Studies in Physiology, Diagnosis, and Treatment, edited by C. R. Lam., p. 202, Philadelphia: W. B. Saunders.

Cumming, G. R., and Finkel, F. (1961): Intracardiac Myxoma Involving the Right and Left Atria in a Young Patient, J. Pediat., 58, 559.

Dick, H. J., and MulliN, E. W. (1956): Myxoma of the Heart Complicated by Bloodstream Infection by Staphylococcus Aureus and Candida Parapsilosis, N.Y. St. J. Med., 56(i), 856.

Dollery, C. T., West, J. B., GoodWin, J. F., Hugh-Jones, P., and Wilckens, D. E. L. (1961): Regional Pulmonary Blood Flow in Mitral and Congenital Heart Disease, in Ciba Foundation Study Group No. 8, p. 17, London: J. and A. Churchill.

Dugan, D. J., and Walstad, P. M. (1960): Left Atrial Myxoma: Surgical Cure, Dis. Chest, 38, 429.

Ellis, F. H. Jr., MaNKIN, H. T., and BurChell, H. B. (1958): Myxoma of the Atrium: Successful Surgical Treatment in Two Cases, Med. Clin. N. Amer., 42, 1087.

Fisher, E. R., and Hellstrom, H. R. (1960): Evi- dence in Support of the Neoplastic Nature Cardiac Myxoma, Amer. Heart J., 60, 630.

GoldberG, H. P., GlenN, F., DOTTER, C. T., arf STEINBERG, I. (1952): Myxoma of the Left Atrium. Diagnosis made during Life with Operative and Pos Mortem Findings, Circulation, 6, 762.

Goodwin, J. F., Stanfield, C. A., Steiner, R. E⿱ Bentall, H. H., SAYed, H. M., BloOM, V. R., arff Bishop, M. B. (1962): Clinical Features of Leff Atrial Myxoma, Thorax, 17, 91 .

GoodwiN, J. F. (1963): Diagnosis of Left Atri\$ Myxoma, Lancet, i, 464.

GraVier, J., and SCHLIENGER, R. (1960): Raport ive L'Angiocardiographie Dans Le Diagnostic De Tumeurs De L'Oreillette, J. Radiol. Electrol., 41 273.

HaRvey, J. C. (1957): Myxoma of the Left Auricle. Ann. intern. Med., 47, 1067.

HusteN, K. (1922): Uber Tumoren und Pseude tumoren des Endocards, Beitr. path. Anat., 71, 132.

KIDD, B. S. L., CARSON, D. J. L., and LAMONT, E. \& (1961): Intra-atrial Sarcoma, Brit. med. J., ii, 1476

LEKISCH, K. (1957): Myxoma of the Left Atriumgo Report of a Case, Ann. intern. Med., 46, 982.

Levinson, J. P., and KINCAID, O. W. (1961): Myxom of the Right Atrium Associated with Polycythaemia Report of Successful Excision, New Engl. J. Med. 264, 1187.

MacGegor, G. A., and Cullen, R. A. (1959): The Syndrome of Fever, Anaemia, and High Sedimenta tion Rate with an Atrial Myxoma, Brit. med. J., 991.

Mills, P., and Philpott, M. (1951): Myxomas of Heart with Neurological Signs, Brit. Heart J.o1\$ 115.

Prichard, R. W. (1951): Tumours of the Herit. Review of the Subject and Report of One Hundred and Fifty Cases, Arch. Path., 51, 98.

Ravid, J. M., and SACHS, J. (1943): Tumours of the Heart. With a Report of a Primary Fibromyx@ sarcoma of the Left Auricle and the Pulmonar Vein Associated with Multiple Tumours of the Mesentery and Alimentary Tract, Amer. Heart 26, 385 .

Siggillino, J. J., Crawley, C. J., Clauss, R. H. ReED, G. E., and TICE, D. A. (1963): Myxoma of the Right Atrium with Polycythaemia, Arch. intern. Med., 111, 178.

SteinberG, I., DOTTER, C. T., and GlenN, F. (1953)를 Myxoma of the Heart. Röentgen Diagnosis During Life in Three Cases, Dis. Chest, 24, 509.

Straus, R., and Merliss, R. (1945): Primary Tumotur of the Heart Arch. Path., 39, 74.

THOREL, C. (1903): Geschwulste des Herzens, Ergeb allg. Path. path. Anat., 9, 901. 